\title{
Comparing the effectiveness of two real-time train rescheduling systems in case of perturbed traffic conditions
}

\author{
S. Wegele ${ }^{1}$, F. Corman ${ }^{2} \&$ A. D' Ariano ${ }^{2}$ \\ ${ }^{1}$ Institute for Traffic Safety and Automation Engineering, \\ Technical University of Braunschweig, Germany \\ ${ }^{2}$ Transport and Planning, Delft University of Technology, The Netherlands
}

\begin{abstract}
Rescheduling train traffic in a busy and complex railway area is a challenging task, partly because of the high number of constraints to be taken into account, and partly because of the many variables involved. Currently this task is performed almost exclusively by human traffic operators. Previous attempts to provide an automated decision support system have been limited to identifying and solving train conflicts locally. Recently innovative dispatching support tools have been presented that are able to cope with large (real-time) timetable perturbations, such as train delays and their propagation. However, there is a lack of computational studies that underline their additional practical value.

In this paper we compare two advanced support systems for real-time rescheduling of train operations, developed for the German and Dutch railway networks. The research aim is to establish a bench mark for future co-operation and exchange of innovative solutions. A common test case from the Dutch railway network, the dispatching area between Utrecht and Den Bosch, and disturbed traffic conditions are studied to evaluate the two dispatching support tools in terms of delay minimization. Since these tools make use of different mathematical optimization techniques for the computation of running times and train sequences, a detailed comparison of the proposed rescheduling solutions is provided. The use of railway capacity is illustrated in order to enable an easy and fast detection of the conflicts between the trains running in the network and to get precise information about their resolution by the different rescheduling techniques.
\end{abstract}

Keywords: decision support, computer techniques, dynamic train regulations. 


\section{Introduction}

Railway traffic is currently operated according to a timetable designed months in advance. However, during operations unforeseen events generate conflicts or even deadlocks in the network and rescheduling is therefore needed to obtain feasibility and to minimize the propagation of delays. To cope with this problem, dispatchers have to take rescheduling decisions on the basis of their experience or of predetermined rules to manage disturbed traffic situations. As a consequence, rescheduling decisions may be sub-optimal since there is a lack of information about the impact of these decisions on the future evolution of traffic flow.

Dispatching support tools have been proposed to help dispatchers in performing their task more effectively. Such tools perform a thorough search in the space of solutions, analyzing the effects of multiple rescheduling decisions and computing feasible solutions (i.e., conflict-free and deadlock-free schedules) in a short time.

This paper presents a comparative study to assess the effectiveness of two decision support tools for optimal rescheduling of train operations, developed for the Dutch and German railway networks (see respectively D'Ariano [1] and Wegele et al. [2]). A comparison between the tools is achieved thanks to the availability of railway instances in digital format in order to establish a bench mark for future co-operation and exchange of innovative solutions.

The paper is organized as follows. Section 2 describes briefly the two dispatching support tools, identifying similarities and differences. Section 3 presents the computational experiments on a common test case, a Dutch railway dispatching area, for small delay situations. The results obtained by the two tools are compared in terms of delay minimization. Section 4 then gives conclusions on the performance comparison between the two tools.

\section{Dispatching support tools}

Existing support tools for real-time train rescheduling can be characterized by the general architecture shown in Figure 1. Reliable and accurate data about the positions and speeds of the trains running in the studied area, as well as the infrastructure status and deviations from the timetable, are used in order to evaluate a number of scheduling options, that comply with the constraints of safety system and the current dynamics of trains. The best solution found is reported to the dispatcher for approval and practical implementation.

The dispatching task is based on the following rescheduling techniques: adjusting the running time of trains in open track corridors (re-timing), changing the train sequences at merging and crossing points (re-ordering), modifying the train routes (re-routing) or even cancelling train services in case of large delays.

The schedule re-optimization makes use of the above rescheduling techniques and considers usually an explicit objective function, such as the minimization of a weighted sum of delays or other functions based on train priorities. In the current dispatching practice, straightforward rules like first-come-first-served are adopted since these are simple to be implemented while in the related literature advanced 


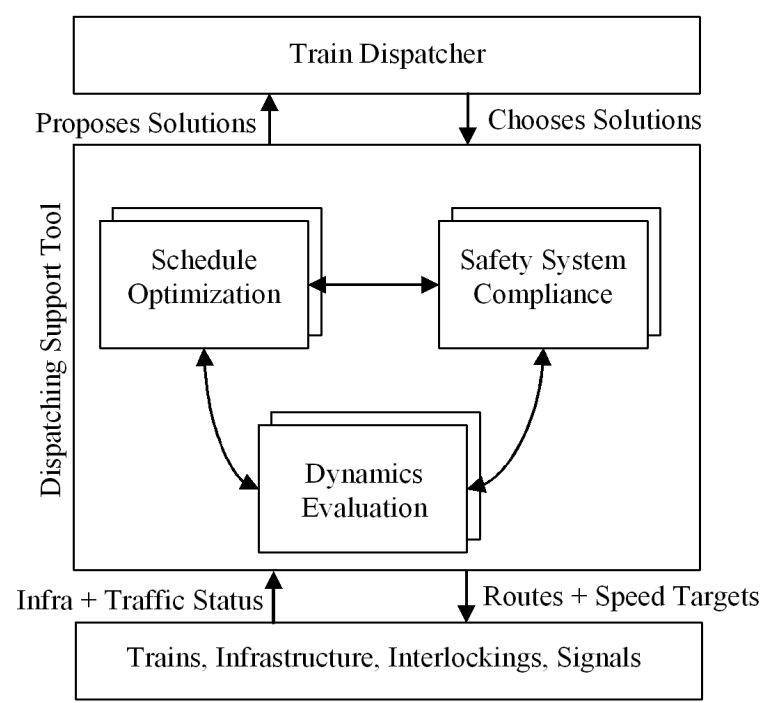

Figure 1: Architecture of a general dispatching support tool.

heuristics and metaheuristics have been developed with more promising results. However, advanced algorithms still have to be implemented in a dispatching support tool connected with actual track occupation and release data in order to automatically detect delays, headway and route conflicts.

The precise computation of train dynamics, needed to forecast the traffic flow in the network, can be performed by different degrees of accuracy, for instance by solving the motion differential equation or using approximated expressions. Note that safety systems and operating rules may vary greatly from one country to another, influencing the way traffic flow is managed.

We next describe briefly the Dutch tool ROMA and the German tool GADis.

\subsection{ROMA system}

The ROMA (Railway Optimization by Means of Alternative graphs) system [1] is a laboratory dispatching support tool that integrates advanced OR techniques to solve global conflict detection and resolution problems with a precise modeling of the train dynamics and coordination of train speeds (see Figure 2).

In the ROMA system, the train traffic flow is modeled as a job shop scheduling problem and represented by means of alternative graphs. Innovative train scheduling and rerouting algorithms have been developed for the resolution of complex global conflict detection and resolution problems. In this paper, we make use of a state-of-the-art branch and bound algorithm which includes static and dynamic implication rules enabling to speed up the computation, advanced initial heuristics, empirical branching rules and a fast and tight lower bound [3]. 


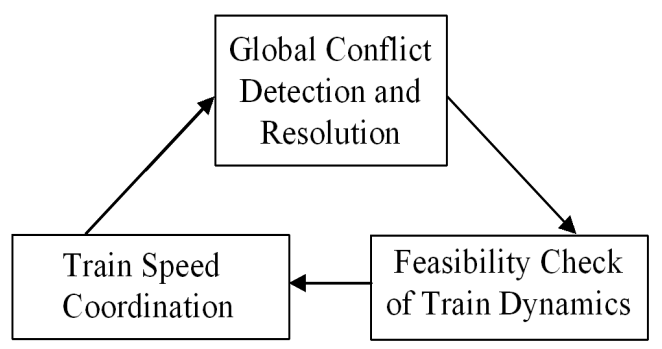

Figure 2: Core structure of the ROMA system.

After a feasible (optimal) schedule has been computed by the branch and bound algorithm, a train speed coordination procedure based on blocking time theory (see e.g. Hansen and Pachl [4]) checks whether the minimum required headway distance between consecutive trains is respected. Otherwise, the train dynamics are precisely re-computed by means of acceleration and braking tables and according to the Dutch signaling system NS54 and the ATB train protection system [5].

\subsection{GADis system}

The GADis (Genetic Algorithm Dispatching) system [6] bases the optimization of rescheduling decisions on the following hybrid optimization method. Trains are inserted one by one and the resulting decision tree of train sequences and routes is explored by a branch and bound search. The resulting solution quality depends on the insertion order of trains and the penalty estimation function used during the search. After inserting all trains, a local optimal solution is obtained. The insertion order of trains and the penalty estimation function are then varied by evolutionary genetic algorithms in order to get another local optimum, which may eventually be better than the already calculated one (see Figure 3).

To enable the GADis system to simulate a Dutch network, the infrastructure had to be translated into the RailML-based format. Since the cab signalling and train

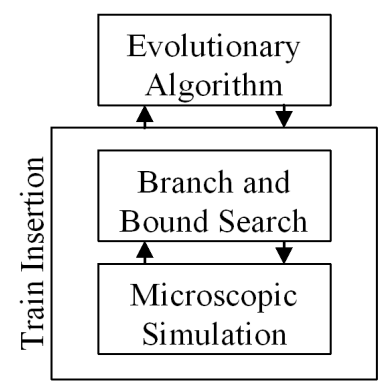

Figure 3: Core structure of the GADis system. 
protection system has implemented the default German PZB90 (Punktförmige Zugbeeinflussung, point-wise train protection) system combined with the LZB (Linienzugbeeinflussung, continuous train control) system, the block sections of the Dutch ATB (Automatische Treinbeinvloeding, automatic train protection) system were too short for PZB90 distant signals. The ATB system had therefore to be converted into the LZB system and the position of signals has been modified accordingly, i.e., each block section is around one $\mathrm{km}$ long.

\subsection{Overview of the main differences between the two tools}

Figure 4 gives a schematic description of some important features characterizing the two dispatching support tools presented in this paper.

ROMA is applied to the Dutch three-aspect fixed block signaling system. If the minimum required headway distance between two consecutive trains is not respected, the following train encounters a yellow or even red signal aspect and has to start deceleration until its speed is reduced to $40 \mathrm{~km} / \mathrm{h}$ or $0 \mathrm{~km} / \mathrm{h}$ respectively. Nevertheless, if the block section ahead becomes available, the signal is cleared and the following train may re-accelerate.

In the current implementation of GADis, the signaling and train protection systems are simplified as follows. Each train checks the aspect of the current signal at the beginning of the braking curve; if the next signal aspect is red, the train will stop. In other words, a train is committed to stop whenever its distance from a red signal aspect is equal to the actual braking distance. Due to this assumption, the train performs a stop and re-acceleration from standstill even if the red signal aspect is cleared during braking. As a result of the different modeling of the train dynamics in case of varying signaling aspect, GADis enables the trains to use the railway infrastructure capacity more generously, especially at short block sections.

\begin{tabular}{|c|c|c|}
\hline System & ROMA & GADis \\
\hline Train dynamics & $\begin{array}{c}\text { Tables of acceleration and } \\
\text { braking given by ProRail, } \\
\text { additional resistances and } \\
\text { gradients are not included }\end{array}$ & $\begin{array}{c}\text { Microscopic solution of } \\
\text { differential equations } \\
\text { for train as a mass point, } \\
\text { acceleration and resistance } \\
\text { curves are considered for } \\
\text { locomotives and wagon, } \\
\text { gradients and resistances }\end{array}$ \\
\hline $\begin{array}{c}\text { Train protection } \\
\text { and signaling } \\
\text { system }\end{array}$ & $\begin{array}{c}\text { ATB and Dutch block-based } \\
\text { signaling system NS54 }\end{array}$ & $\begin{array}{c}\text { LZB and PZB90 without } \\
\text { taking into consideration } \\
\text { the position of balises }\end{array}$ \\
\hline $\begin{array}{c}\text { Optimization } \\
\text { method }\end{array}$ & $\begin{array}{c}\text { Global train reordering by a } \\
\text { branch and bound algorithm }\end{array}$ & $\begin{array}{c}\text { Heuristic train reordering } \\
\text { by genetic algorithms }\end{array}$ \\
\hline
\end{tabular}

Figure 4: Comparing features of the two dispatching support tools. 


\section{Experimental results}

This section presents a common test case from a complex dispatching area in the Netherlands. For given traffic conditions, the two dispatching support tools are compared in terms of train dynamics and train rescheduling solutions. The use of railway capacity is also illustrated in order to enable an easy and fast detection of train conflicts and to get precise information about their resolution by the different rescheduling techniques.

\subsection{Test case description}

The GADis and ROMA systems were tested on the Dutch dispatching area between Utrecht and Den Bosch. The network consists of a corridor around $50 \mathrm{~km}$ long with two main tracks, seven passenger stations, a dedicated stop for freight trains and several merging and crossing points along the network. Figure 5 shows a scheme of the dispatching area, where the station of Utrecht is just out of the left border, the Geldermalsen station is roughly in the middle of the area and the Den Bosch station is at the right end. A layout, at a similar scale, will also be adopted for the blocking time plots presented in this section.

The computational experiments were made on the basis of a cyclic timetable for year 2007, that is characterized by heterogeneous traffic (intercity trains and local train services) and includes freight trains directed to Germany. In peak hours, 26 trains are scheduled in Geldermalsen and up to 40 trains in Den Bosch.

The timetable includes recovery times and buffer times between the train routes. Recovery times can be utilized to recover from delays by running at maximum speed and shorten the scheduled train stops to a minimum dwell time. Buffer times prevent or reduce the delay propagation to other trains.

\subsection{Comparison of train dynamics}

Since the two dispatching support tools compute the train dynamics with a different degree of approximation and use different signaling and train protection systems, we first compare the simulated schedules obtained in absence of delays.

Figure 6 shows the blocking time plots by the two tools for the scheduled train sequence in the timetable. For both tools, the (total) travel time of all trains within

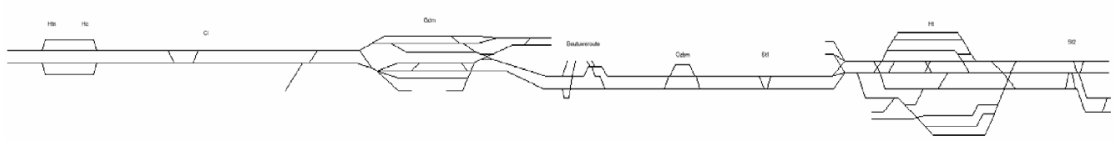

Figure 5: Dispatching area between Utrecht (left side) and Den Bosch (right side). 


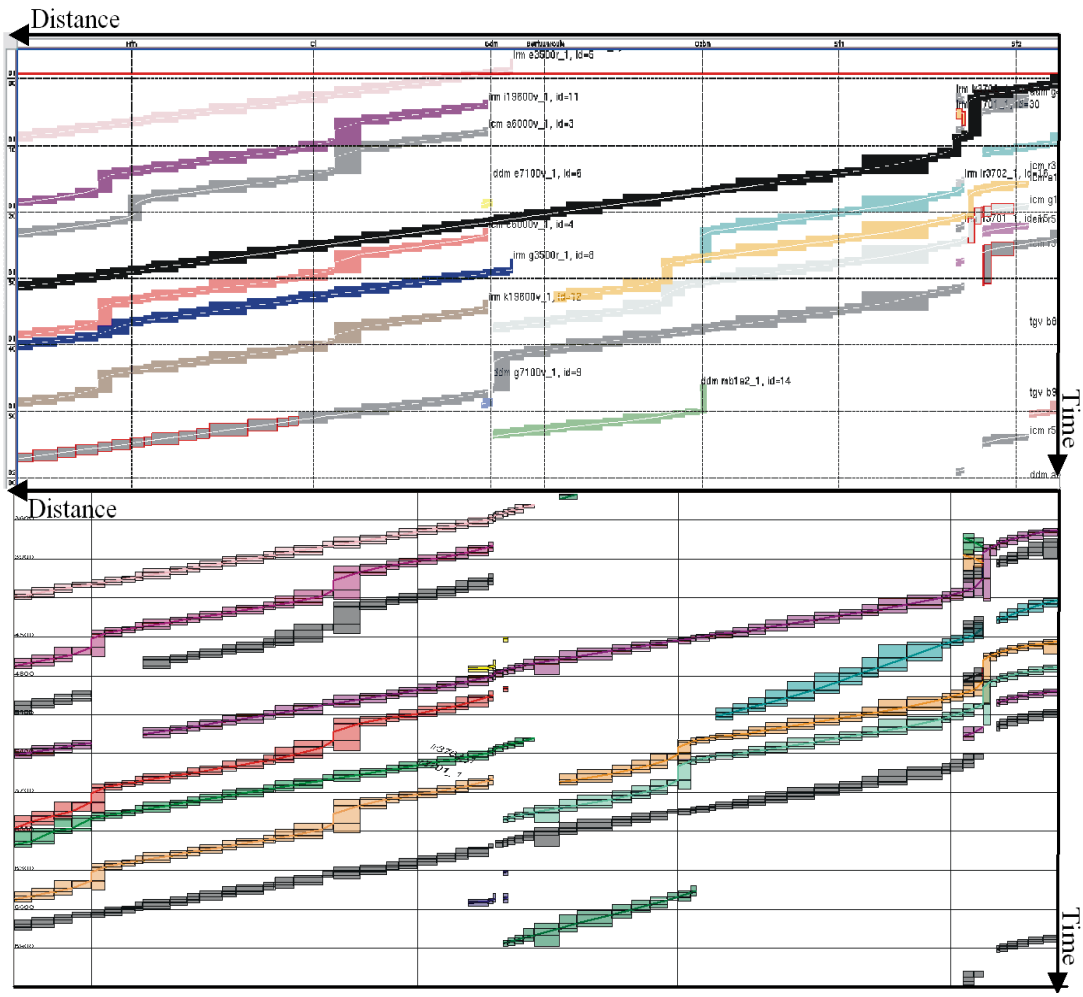

Figure 6: Timetable solution computed by the two dispatching support tools (upper graph: GADis, lower graph: ROMA).

the studied area is around 33000 seconds. In the unperturbed situation, ROMA has found no output delay. On the other hand, GADis reports headway conflicts. In the latter case, dispatching trains according to the timetable results in 68 seconds of total output delay. These are due to the shorter block signal spacing assumed and the automated booking of some train routes induced by LZB system.

We now consider a timetable perturbation generated by postponing the entrance of two trains in the network. For the given delay scenario, we compute a feasible schedule by each dispatching support tool by scheduling trains according to the sequence fixed in the timetable. In the blocking time plots of Figure 7, each of the two postponed trains, indicated as 2 and 4, has got an input delay of 600 seconds and causes a domino effect of delay propagation in the network. In fact, two trains, called 1 and 3, are more affected by trains 2 and 4 . For those four trains, the scheduled train sequence is 2-4-3-1.

If the train sequence is fixed as in the timetable, in the ROMA solution the total travel time is 35810 seconds, the number of disturbed trains is 11 and the total output delay is around 3000 seconds. GADis presents a different situation with 


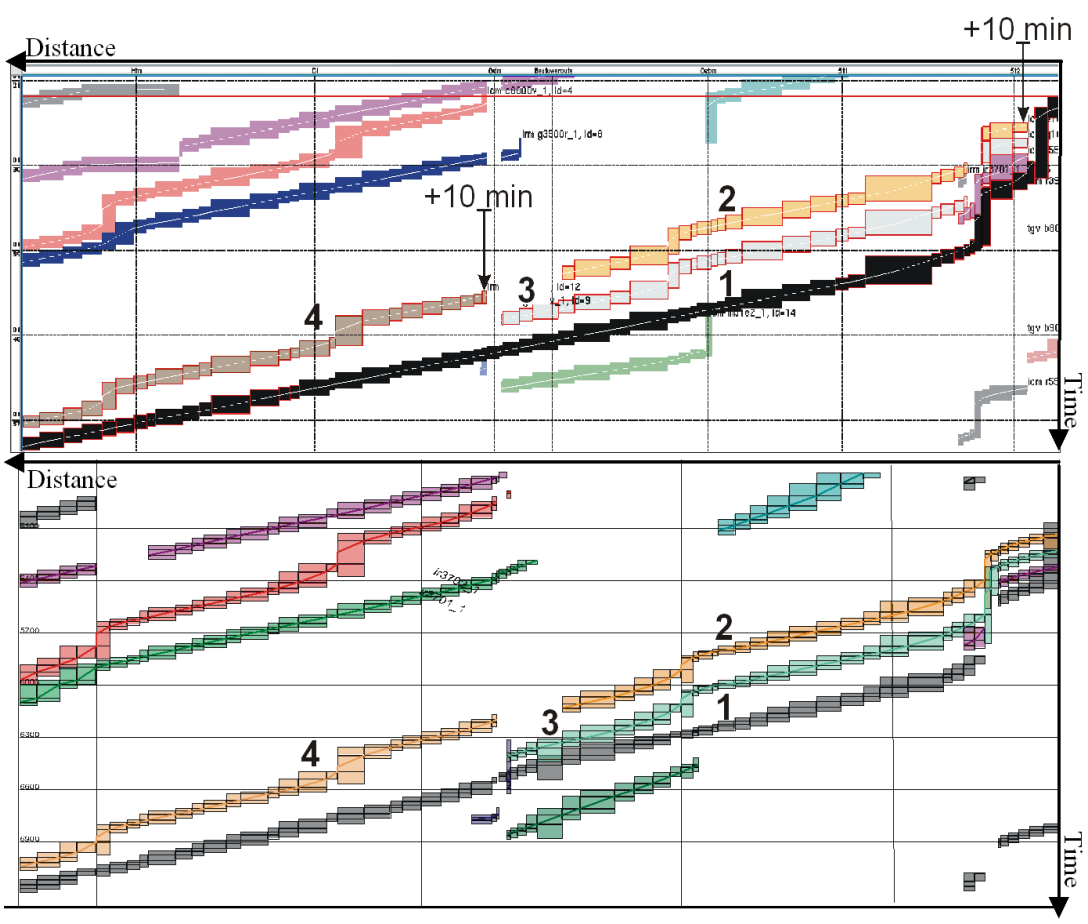

Figure 7: Timetable sequence (no reordering of trains) for the given delay scenario (upper graph: GADis, lower graph: ROMA).

36030 seconds of total travel time, 9 disturbed trains and around 3700 seconds of total output delay. The larger number of delays is again explained by the different signaling and train protection systems and computation of train dynamics, resulting in an unequal use of railway infrastructure capacity.

\subsection{Comparison of train rescheduling solutions}

The delay scenario introduced in the previous subsection has been used as an input to find an optimized solution by both dispatching support tools. A comparison between the rescheduling solutions is proposed in this subsection.

Figure 8 shows the rescheduling solution found by each dispatching support tool using its corresponding optimization algorithms and refers to the four most delayed trains. Specifically, GADis proposes the train sequence 3-2-4-1 and a total travel time of 34070 seconds, while ROMA suggests 3-1-2-4 and a total travel time of 34370 seconds. In terms of total output delays, GADis generates 1250 seconds and ROMA only 1010 seconds. From the obtained values, it is interesting to compare the travel time against the output delays. Since ROMA is able to use the recovery times more effectively, its rescheduling solution exhibits less output delays but 


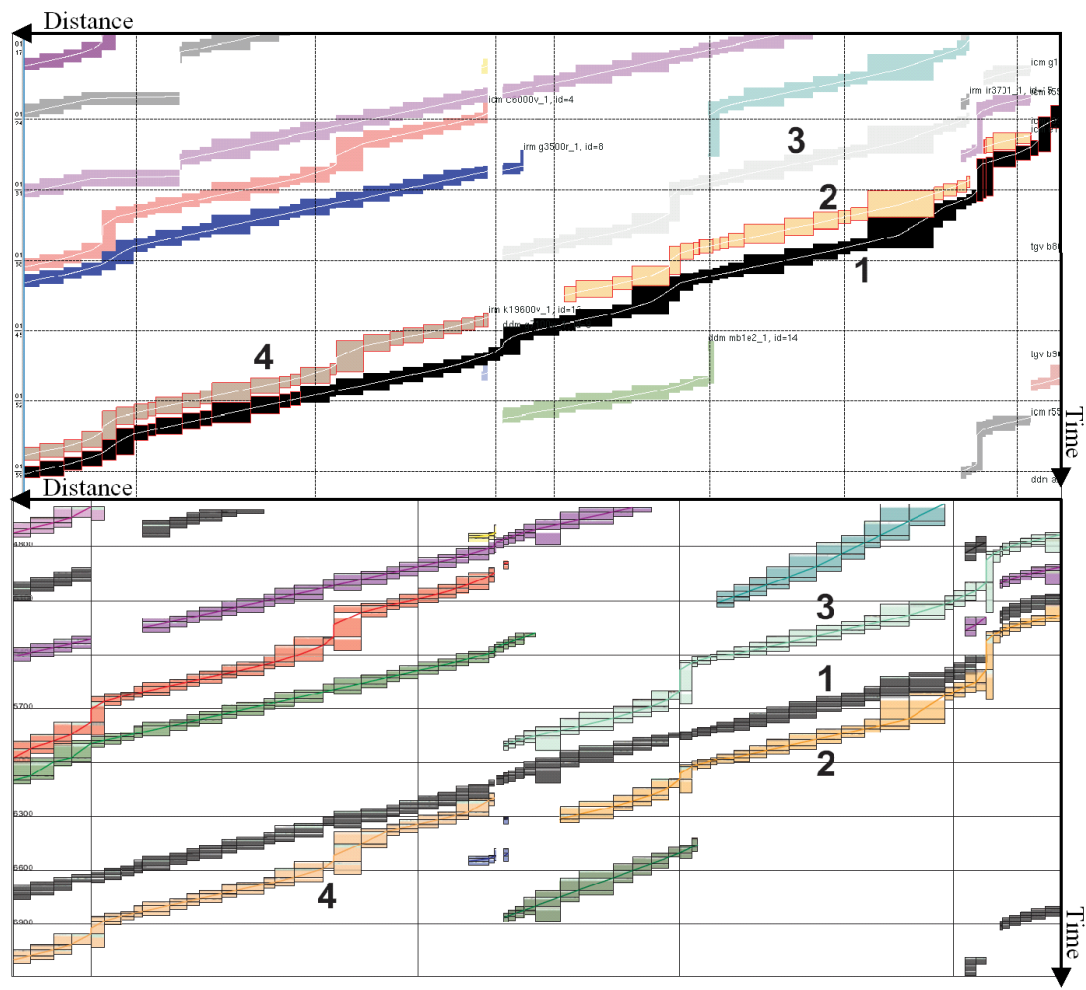

Figure 8: Solution of the rescheduling algorithms for the proposed delay scenario (upper graph: GADis, lower graph: ROMA).

larger travel times than GADis. In general, both tools improved significantly the solution obtained in the previous subsection.

The GADis solution contains the following differences compared to the train orders fixed in the timetable. Trains 2 and 4 are now scheduled after train 3 and before train 1 , even if the latter train has to stop repeatedly due to headway conflicts to preceding trains. This effect is more evident in Den Bosch (right side of Figure 8 , upper graph) that is a rather complicated and densely occupied area.

ROMA also changes the train sequence compared to the timetable. The blocking time graphs of trains 2 and 4 are shifted after those of trains 3 and 1 so that the delay propagation is minimized. In fact, the total output delay is even less than the sum of the input delays of trains 2 and 4 . The existing network capacity reserve is thus exploited by ROMA such that the overall traffic flow remains stable.

\section{Conclusions}

Two existing dispatching support tools have been tested on a real-world case study from the Dutch railway network. The dispatching solutions obtained by the two 
tools and their rescheduling algorithms have been compared in terms of detailed blocking time plots, output delays and travel time spent. The solutions present a different computation of train dynamics even for unperturbed traffic conditions because of different modeling of the signaling and train protection systems.

A more balanced comparison of the performance of the two dispatching support tools can be achieved if the tools adopt the signaling and train protection systems with short headways between trains, especially in the neighborhood of stations.

Despite the above limitations, demanding convertible infrastructure and digital timetable and rolling stock data should be enforced since this would provide a common setup for the evaluation of advanced rescheduling algorithms. To this end, international standard platforms, such as RailML, should be used to stimulate transferability of scheduling and dispatching support tools between different railway networks and countries.

\section{References}

[1] D’Ariano, A., Improving Real-Time Train Dispatching: Models, Algorithms and Applications. TRAIL Thesis Series T2008/6, The Netherlands, 2008.

[2] Wegele, S., Slovák, R. \& Schnieder, E., Real-time decision support for optimal dispatching of train operation. In: $C D$-ROM Proceedings of the 2 nd International Seminar on Railway Operations Modelling and Analysis, Hannover, Germany, 2007.

[3] D’Ariano, A., Pacciarelli, D. \& Pranzo, M., A branch and bound algorithm for scheduling trains on a railway network, European Journal of Operational Research, 183(2), pp. 643-657, 2007.

[4] Hansen, I.A. \& Pachl, J., Railway timetable and traffic: Analysis, Modelling and Simulation, Eurail Press, Germany, 2008.

[5] D’Ariano, A., Pranzo, M. \& Hansen, I.A., Conflict resolution and train speed co-ordination for solving real-time timetable perturbations, IEEE Transactions on Intelligent Transportation Systems, 8(2), pp. 208-222, 2007.

[6] Wegele, S., Slovák, R. \& E. Schnieder, E., Automatic dispatching of train operations using a hybrid optimisation method. In: CD-ROM Proceedings of the 8th World Congress on Railway Research, Seoul, Korea, 2008. 